QL 426 A1L 34 1869 MOLL 


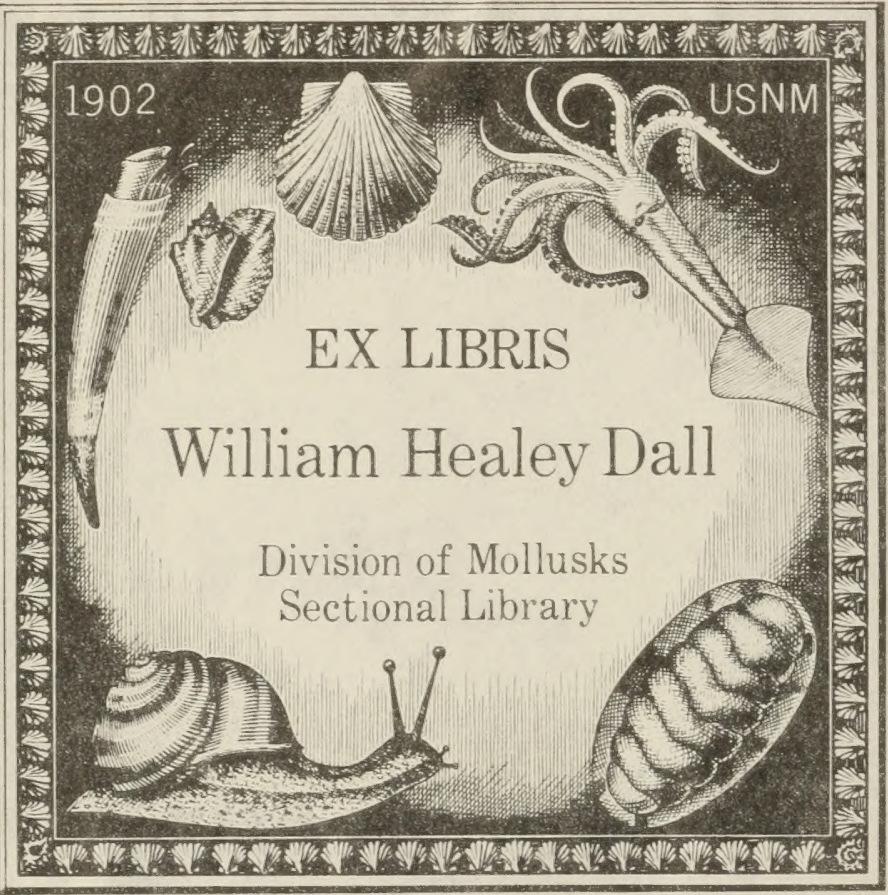



Division of Mollusks

Sectional tibrary 



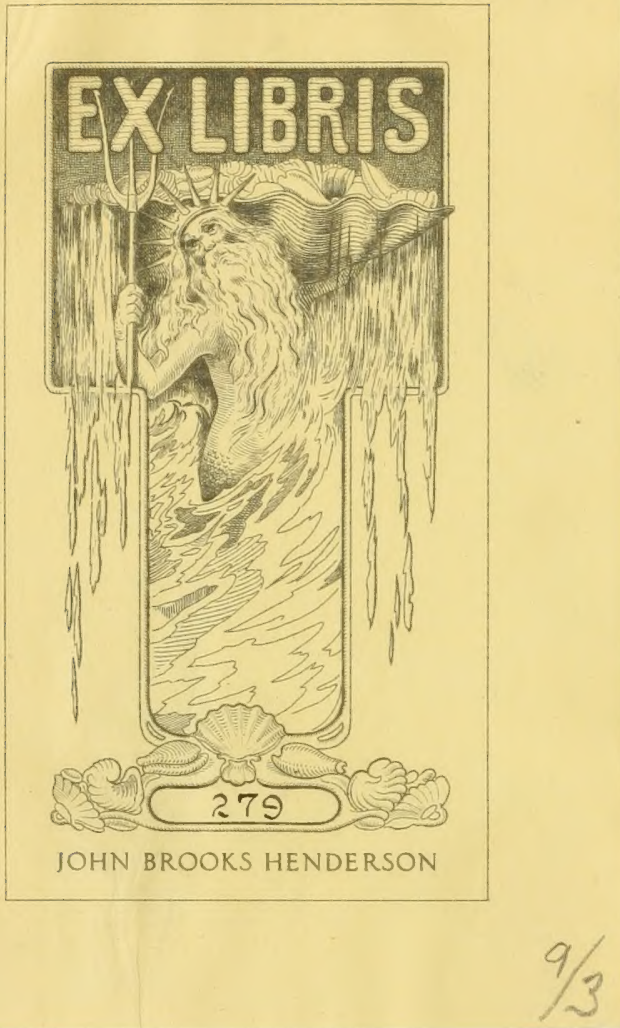


\section{CATALOGUE}

DES

\section{IOLLUSQUES TERRESTRES ET FLUVLTILES}

OBSERVÉS

AUX ENVIRONS DE JAULGONNE (AISNE). 



\section{CATALOGUE}

IIES

\section{HOLLUSOURS TERRESTRES ET FLUVITILES}

OBSERYÉS

AUX ENVIRONS DE JAULGONNE (Aisne)

PAR

MII. CII. LALLENIANT ET G. SERVAIN.

AOUT 1869.

Paris,

HUPRIMERIE ET LIBRMIRIF IDE NIme Ve BOUCHARD-IIUZARI, RUE DE L'ÉPERON, 5. 


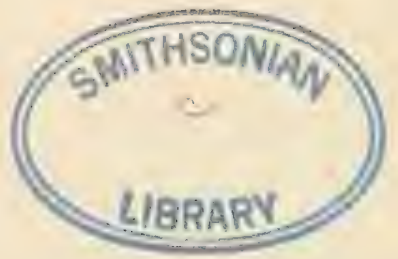


Jaulgonne, donl nous entreprenons l'histoire malacologirge, est un bourer du département de l'Aisne, situé sur le bord de la Marne. à 12 kilumètres environ an N. E. de Chiteau-Thierry.

Ce bourg, ansis dints une jolie villée, est entouré, a l'est et à l'ouest, par de hautes collines couronnées de forèts; un ruisseau, alimenlé par de nombreuses sources, le traverse dans tonte sa longueur et entretient dans les parties basses une humidité constante très-favorable a la population malacologigue dont nous nous faisons en ce moment les historiens.

Tos recherches anx environs de 10 eharmant pays unt embrassé toute la vallée de la Marne jusyu'au delit de Barzy, ainsi que tous les coteaux, la plupart rouverts de riches vignobles; alles se sont étendurs mome dans la petite vallere d'Argent-

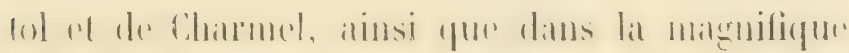


forêt de Riz, où nous avons trouvé en abondance des espèces de toute sorte.

Les mollusques dont nous présentons le catalogue proviennent tous, à l'exception de quelquesuns, du centre alpique.

Celte faune est donc une faune d'acciimatation étrangère au pays.

Nous prions nos savants et excellents amis, MI. J. R. Bourguignat et J. Mabille, de vouloir bien recevoir l'expression de notre profonde gratitude pour leur obligeance, leur affabilité, et surtout pour l'empressement qu'ils ont bien voulu mettre à vérifier l'exactitude de nos déterminalions et la véracité de nos espèces.

Paris, aoùt 1869 .

GH. L. - G. S. 


\title{
MOLLUSCA GAS'TEROPODA.
}

\section{GLSTEROPODA INOPERCLLATA.}

\author{
S 1. PULMONACEA.
}

\author{
ARIONIDE.
}

\author{
G. ARION.
}

\section{ARION RUFUS。}

Limax rufus, Limnaus, Syst. nat. (éu. X), p. 652, 17088. Arion empiricorum, Férussac, Hist. Holl., p. 60, pl, I, f. $3,1819$.

Arion rufus, Michaud, Compl. a Drap., p. 5, 1851.

Lspèce très-abondante sur les bords de la Marne, dans les parlies ombragées des bois, notamment dans la foret de Riz. 


\title{
ARION HORTENSIS:
}

Arion hortensis, Férussac, Hist. Moll., p. 63, pl. II, f. $4-6,1819$.

Commun dans les jardins, sous les pierres, au pied des plantes. - Ravin de Cliarmel, sur les troncs des arbres.

\section{ARION BOURGUIGNATI.}

Arion Bourguignali, Mabille, Archiv. malac., I, P. 14, déc. 1867, et in Rev. et mag. zool., p. 159, 1868.

Sous les pierres, près du Charmel.

\section{LIMACIDA.}

\author{
G. LIMAX.
}

\section{IIMAX VARIEGATUS.}

Limas variegatus, Draparnaud, Tabl. Moll., p. 103, 1801, et Hist. Moll. France, p. 127, 1805.

Dans les caves, à Jaulgoune.

\section{IIMAX CINEREO-NIGER.}

Limax rincreo-niger, Holf, in Sturm, Teulsch. Fauna, Wurmer, fasc. 1, 1805. 
Sous les bois morts, dans les parties humides de la forêt de Riz.

\section{IIMAX CINEREUS.}

Limas cinereus, Mïller, Verm. IIist., II, p. 5, 177 .

Dans les bois des environs de Jaulgonne, de Barzy, etc.

- Dans les jardins, au pied des murs.

\section{IIMAX ARBORUM.}

Limax arborum, Bonchard-Chantereaux, Moll. I'as-ileCalais, p. 28, 1858.

Espèce commune sur les arbres dans la forêt de Riz.

\section{IMMAX AGRESTIS.}

Limax agrestis, Linnous, Syst. nat. (éd. X), 1. (i52, 1758.

Très-abondante dans les jardins et les prairies.

$$
\text { G. KRYNICKILLUS. }
$$

\section{KRYNICKIYIUS BRUNNEUS.}

Limax brunneus, Draparnaud, Tabl. Moll., f. I0'. 1801, ef Hist. Moll. France, p. 128, 1805. 
Lirynickillus brunnens, J. Mabille, Ireh. malac, I, p. 47, déc. 1867 .

Bords de la Marne, sous les pierres el les morcearix de bois.

\section{HELICID N.}

G. VITRINA.

\section{VITRINA MAJOR.}

Helicolimas major, Férussac (père), Lssai llćth. conch., p. $45,1807$.

Vitrina major, C. Pfeiffer, Deutschl. Holl, I, p. 17 (en note), 1821.

Cette espèce habite sous les feuilies, an pied des arbres, is Argentol.

\section{VITRINA PELLUCIDA.}

Ilelix pellucida, HHüller, Verm. IIist., II, p. $150,1774$. Vitrina pellucida, Giortner (1), Conch. Wetter., p. 5', 1815.

Sur les coteaus du bois de Jouarre. - Espèce peu rommune.

(1) Nou Vitrina pellucida de Draparnaud, Ilist. Moll., f. 109, pl. vut, f. $33-35,1805$, qui est une espèce différente. 


\section{G. SUCGINEA.}

\section{SUCCINEA PUTRIS.}

Ileir putris, Limnaus, Syst. nat. (éd. X), 1, 774, 1758. Succinca putris, de Blaimville, in Dict. sc. nal., vol. LI, p. 244, tab. xxxv, f. 4, 1827.

Dans les lieux humides ou marícageux, sur les plantes aux bords de la Marne, dans les prairies de Chartives, et dans presque tous les alentours de Jaulgonne et de Charmel.

\section{SUCCINEA PFEIFFERT.}

Succinea I'feifferi, Rossmissler, Iconogr., I, P., 96, f. $46,185 \mathrm{~s}$.

Ilabite avec la précédente, mais nolamment ì Mezy, sur les bords de la llarne, et sur les plantes ayuatiques vers l'embouchure du Surmelin.

\section{SUCCINEA MAIMILATA.}

Succinea mamillata, J. Malille, Ilist. Moll. terr. flur. bassin parisien (sous presse), 1869.

Espece assez rare. Cá el lit dans les prairies de la llarne. 


\section{SUCCINEA BAUDONI.}

Suscinca Baudoni, Bonryuigmal, in Amén. malac., I, p. 159, pl. x, f. 1-5, janv. 1856.

linvirons de Jangonne, dans les parties un peu sèches des prairies. - Espèce rare.

\section{SUCCINEA OBLONGA.}

Succinea ohlonga, Draparnaul, Tahl. Holl., p. 56 , 1801, et Hist. Mioll. France, p. 5ै6, pl. III, f. $24-25,1805$.

Sur les plantes, le long de la roule de Jaulgonne a Tréloup. - Prairies de la Marne.

\section{SUCCINEA ARENARIA.}

Succinea arenaria, Bouchard-Chomtereaux, Catal. Moll. Pas-de-Calais, p. 100, 1858.

Dans les prairies aus environs de Jaulgonne, de Barzy, de Charmel, etc.

\section{G. ZONITES.}

\section{ZONITES FUIVUS.}

IIelix fulva, Mïller, Verm. Ilist., II, p. Ӟ6, 177 1. Zonites fulvas, Moquin-Tandon, Hist. Moll., II, 1. 67 , lab. vili, f. 1-4, 1853.

Sous les pierres el les délritus au moulin d'Ar- 
gantol, et pres da marais du moulin lamnay, ainsi que dans les parlies humides de la forêt de Riz.

\section{ZONITES LUCIDUS.}

Holix lucila, Drapurnmed, Tabl. Mol!, p. 96, 1801, et Hist. Moll. France (exclus. desc.), tab. vir, f. $25-25,1805$.

Zorrites lucidus, Bourguignut, Cat. coq. d'Orient, in Voy. à la mer Morte, p. 8 (en note), 1855 .

Sous les pierres, le long des murs, à Jaulgonne.

\section{ZONITES SEPTENTRIONATIS.}

Zonites septentrionalis, Bourguignal, g. Zonites, 1869. p. $13,1869$.

Lelourneux, Cat. Moll. Vendée, p. 13,18 .

Sous les détrilus, le long des murs de Jaulgonne.

\section{ZONITES CELTARIUS.}

Ilchs cellaria, Jü̈ller, Verm. Ilist., II, p. 58, 1774. Zonites ceilarius, Gray, in Turtom, Shells Brit., p. 170, 1840.

Sous les pierres, les bois pourris, dans la forêt de liz; dians celle de l'Ilôtel-Dieu, ainsi qu'aux alentours d'Argentol, et au pied des murs de Jaulgonne.

\section{ZONITES ALLIARIUS.}

IIelix alliaria, Willer, in Ann. phil., vol. VII, N. 579 , 1829. 
unites alliarius, Gray, Nan. Shells Brit. 'Turton, p. 168, tab. Iv, f. $59,1840$.

Dans la forêt de Riz.

\section{ZONITES NITENS.}

Helix nitens, Gmelin, Syst. nat., p. 5655, 1788, et Michaud, Compl. à Drap., p. 44, pl. xv, f. $1-5,1851$.

Zonites nitens, Bourguignal, Cat. coq. Orient, in Voy. a la mer Norte, p. 8 (en note), 1835.

L'Aulnay, sous les pierres, sous les feuilles morles, dans les lieux humides. - Ravin de Charmel. - Au pied des murs de Jaulgonne.

On trouve également aux environs de Jaulgonne la variété major de celte espèce, qui a été prise, bien à tort, par Moquin-Tandon, pour le Zonites hiulcus de l'Italie.

\section{ZONITES SUBNITENS.}

Zonites subnitens, Bourguignat, g. Zonites, 1869.

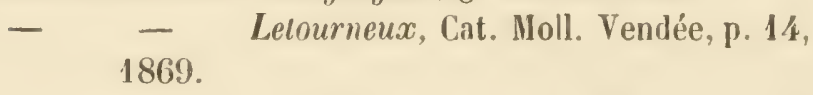

Assez abondante dans les prairies de la Marne et dans la forêt de Riz.

\section{ZONITES NITIDUS.}

Helir nilida, Mïller, Verm. Ilist., II, p. 52, 1774. 
Zonstes nitidus, Morfum-Tandon, Mist. Moll., II, p. 72, 18 ä:

Lispice peu commune. Au bord du petit ruisseau de l'Aulnay, sous les pierres. - Forêt de Riz.

\section{ZONITES PARISIACUS.}

Zoniles parisiacus, J. Mabille, Hist. Moll. terr. Iluv. bassin parisien (sous presse), 1869.

Forêt de Riz. - Rare.

\section{ZONITES NITIDOSUS.}

Helis nitidosa, Férussac, Tahl. sy:1., "1" 214, 1821. Zonites nitidosts, Bourguignat, Mislac. Bret., p. "̈), 1860.

Alluvions du ru d'Argentol et de la Marne.

\section{ZONITES RADIATULUS.}

Ifelix radiatula, Alder, Cat., p. 12, in Newcastl. Trans., I, p. $58,18 \overline{1}$.

Zonites radiatulus, Gray, in Turlon, Man., p. 173, tab. XII, f. 157, 1840 .

Forêt de Riz, sous les détritus, dans lés mouses. Alluvions du ru d'argentol.

\section{ZONITES CRYSTALIINUS.}

Helis cryalallina, Büller, Verm. IIist., II, p. 295, 1774. 
Zonites crystallinus, Gray, in Turton, Man., p. 176 , 1840.

Très-abondante dans les alluvions de la Marne. Ravin de Charmel, sous les feuilles mortes.

\section{ZONITES SUBTERRANEUS,}

Zonites subterraneus, Bourguignal, Amén. malac., I, p. 194, pl. xx, f. 15-18, 1856.

Ravin de Charmel. - Dans les alluvions de la Marne, avec l'espèce précédente.

G. HELIX.

\section{HEIIX POMATIA.}

IIelix pomatia, Linnocus, Syst. nat. (éd. X), p. 771, 1758 .

IIélice des plus abondantes sur toutes les collines, et dans les vignes et les bois.

Sur les coteaux de Barzy, l'on trouve assez fréquemment une variété albescens sans bandes; sur ceux de Charmel, on a recueilli quelquefois des échantillons sénestres.

\section{HELIX ASPERSA.}

IIelix aspersa, Müller, Verm. Ulist., II, p. 3!), 1774. Très-commune partout. 


\section{HELIX NEMORATIS.}

IIclis nemoralis, Limnaus, Syst. nat. (éd. X), p. 775 , 1758.

lspèce très-abondante dans les bois, le long des haies et des cours d'eaux.

\section{HELIX FORTENSIS.}

Helix hortensis, Müller, Verm. Hist., II, [. 52, 1774.

Habite avec la précédente. - Ilélice également trèscommune.

\section{HEIIX ARBUSTORUM.}

Ilelix arbustorum, Linnaus, Syst. nat. (éd. X), p. 771, 1758.

Dans les lieux frais et humides. Bords des ruisseaux et de la Marne.

\section{HELXX FRUTICUR.}

IIelix fruticum, Müller, Verm. Ilist., II, p. 71, 1774.

Dans les haies, sur les arbustes de la vallée de la Marne.

\section{HEIIX INCARNATA.}

Ilelix incarnata, Müller, Verm. Ilist., II, p. 65, 177.4. 
Ravin de Charmel; - ru du Crochet; - lorêt de Riz.

\section{HEIIX CARTEUSIANA.}

Ifelis rarthusianil, Müller, Verm. Mist., II, P. II"̈, 1774.

Très-commune partout, surtout sur les coteaux.

\section{FETIX PIEEIAA.}

Ifclix plebeium, Draparnaud, Hist. Moll. France, p. 105, pl. viI, f. 50, 1803.

Helis plebcia, Michaud, Compl. ì Drap., p. 29, 1831.

Collines de Charmel. - Allurions de la Jarne. Assez rare.

\section{WEIIX SERICEA.}

IIelis sericea, Häller, Verm. IIist., II, P. 62, 177\%.

Collines de Charmel, mais surtout dans les prairies, les lieux frais et humides; sous les pierres.

\section{HEIIX GISPIDA.}

IIelix hispida, Limeces, Syst. nat. (éd. X), p. 774, 17:88. Très-commune, habite avec la précédente. 
Itelis linpicida, Limmerus, siyst. nat. (éd. X), p. 768, 1758.

Sur le tronc des arbres, dans la forcit de Riz; sous les feuilles de licrre, dans le ru du Crochet et dans le ravin de Charmel.

\section{HEIIX OBVOIUTA.}

IIclix obvoluti, Hüller, Verm. Hist., II, p. 27, 1774.

Assez abondante sous les pierres et sous les feuilles mortes dans li forêt de Riz, notamment au ravin d'Argentol et dans le ru du Crochet.

HFXIX PUICEELIA.

Helix pulchella, Müller, Verm. Ilist., II, P. 50, 1774.

Excessivement abondante dans les alluvions de la Marne et de tous les ruisseaur.

\section{IIETIX COSTATA.}

IIclix costata, Müller, Verm. Ilist., II, p. 5I, 1774.

Environs de Charmel; coltaus de Jaulgonne, sous le pierres. - Très-commune également dans les alluvions. 
HEIIX PXGMRA,

IIelis pygmea, Draparnaud, Tabl. Moll., p. 15, 1801, Hist. Moll., p. 114, tab. vili, f. 8-10, 1805.

- $\quad$ Bourguignal, Mloll. nouv. litig. ou peu connus (2e déc.), p. 28, $\mathrm{n}^{0}$ 12, pl. v, f. 1-4, 1865.

Sous les feuilles, dans la forêt de Riz. - Alluvions de la Marne. - Espèce rare, à cause de son extrême petitesse.

\section{GEIIX SERVAINI,}

Celle espèce nouvelle, que notre excellent ami II. J. R. Bourguignat a bien voulu dédier à l'un de nous, appartient au groupe de l'IIelix pygmea. Cette coquille habile, sous les pierres, dans les parties déboisées de la forèt de Riz, du côté de Tréloup.

Voici la description de cette Ilélice nonvelle, d'après M. J. R. Bourguignat:

"Helix Servaini,

"Testa minutissima, late pervio-umbilicala, coma pressa, supra convesa, subpellucida, pallide cornea, in " ultimo anfractu elegantissime lamellicostata (lamella " validx, sicut ciliatæ, presertim in angulo ultimi an" fractus); spira parum convexa; apice obtuso, valido, " levigato, pallidiore; anfractibus \& convexiusculis, re" gulariter crescentibus, sutura impressa separatis; ul" timo anfractu ad partem superiorem subangulato, infra "rotundato ac circa umbilicum leviter turgido; supra " valide, subtus subtilissime lamellicostito, ad aperturam 
« lente descendente; apertura parum obliqua, sub« oblonga, valide lunata; peristomate recto, acuto ac * simplice.

"Coquille très-petite, compriméc, convere en dessus " et pourvue d'un large ombilic en forme d'entonnoir. " Test assez transparent, d'une teinte pâle cornće, of" frant, sur le dernier tour, une série de petites lamelles " ípidermiques, régulières, saillantes, surtont sur la " partie angulense du dernier tour, à ce point qu'elles " ressemblent à des cils. Spire peu convexe. Sommet. " obtus, robuste, lisse, d'une teinte plus pâle. \& tours " peu convexes, ì croissance régulière, séparés pır une « suture assez prononcée. Dernier tour subanguleux vers “ sa partie supéricure, arrondi en dessous, bien qu'assez " renflé autour de la partie ombilicale, légèrement " descendant vers l’ouverture, et sillonné par d'élégantes " lamelles épidermiques saillantes, jrononcées surtout " en dessus et sur la partie anguleuse. Ouverture peu " oblique, fortement échancrée, d'une forme sub« oblongue. Péristome simple droit et aigu.

\section{Haut. 1 millim. \\ Diam. 2}

" Cette nouvelle coquille, que je me fais un plaisir de " dédier à notre ami Georges Servain, est la cinquième a forme, connue en France, du groupe de l'IIelix a pygmaa.

a Les espèces de ce groupe sont, en effet :

" I" L'Ilelix pygmaa (royez ci-dessus pour la synonymie).

" g.'Ilelix Massoti, Bourguignal, Moll. nouv. (2) dicade), p. 50, $n^{\circ} 15$, pl. v, f. 5-8, 186.5 . 
" "o Ilelix micropleuros, Pagel, Dese of a new Ilel. from Nontpellier, in Ann. and mag. nat. Hist. (ser. xiI), p. 131, 18:3, et Bourquignal, Moll. nouv. ( $2^{\mathrm{e}}$ déc.), p. 52, $\mathrm{n}^{\circ} 14, \mathrm{pl}$. v, f. 9-15, $186 \tilde{5}$.

" $4^{\circ}$ Ifelix elachia, Bourguigmal, Moll. nouv. (2 déc.), p. 55, n $15, \mathrm{pl}$. v, f. 14-17, 1863.

"L'Helix Servaini ne peut être, farmi ces espèces, " rapprochée que de l'elachia, dont elle diffère essenticlle" ment par sa taille plus forte; par sa spire plus con" vexe; par son dernier tour seulement lamellé; par son " ouverture plus étroite, plus échancrée, dt... ; surtout " par l'enroulement phus régulier de ses tours, bien " moins rapides que ceux de l'elachia. ")

\section{FELIX ROIUNDATA.}

Helix rolundata, M/iller, Verm. Uist., II, P. $29,1774$.

Espèce abondante, surtout dans la forêt de Riz.

\section{HEIX ACULEATA.}

Helis aculeati, Mhiller, Yerm. Hist., II, p. 81, 1774.

sous les feuilles mortes, dins le ravin de Charmel.

\section{HELIX UNIFASCIATA。}

Helix unifisciata, Poired, Corf flus. el Lerr. de l'tisne of aux environs de Paris, p. 41, avril $\mathbf{1 8 0 1 .}$ 
fus alentours de daulgonne l'on rencontre, notamment sur les coleans, deux formes bien distinctes de cente rspèce : l'une lise, yui est le lype si commun de nos conTrées, puiis une autre it fest strié, y̨ui est une forme alpestre publicée, en 1890, par studer, sous le nom d'Ilelix candidula.

\section{TEEIX STRIATA.}

Helis striata, Dreparnaud, List. Moll. Lrance, P. I06, pl. II, f. 19 (seulement), 1805.

Coleaur de Jaulgonne et de Charmel. - Borits de lit Marne.

\section{HEIIX ERICETORUM.}

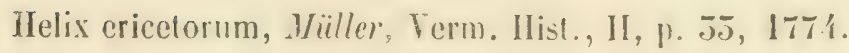

Espèce très-commune partout, surtout dans les en!roits secs, dans les pâtures et sur les coteaux.

\section{FEIIX XAUTA.}

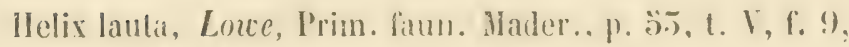
1851.

Ifelix submaritima, Desmoulins, in Rossmeissler, Iconngr. . IX et X, tab. XuIII, f. 575, 1859.

Espece littorale, arcidentellement arclimatée, dans lil plaine de Varenne, vis-ì-vis Jaulgonne. 


\section{G. BULIMUS.}

\section{BUIIMUS MONTANUS.}

Bulimus montanus, Draparnaud, Tabl. Moll., p. 63, 1801, et Hist. Moll. France, p. 74, pl. Iv, f. 22, 1805 .

Coquille assez commune dans la forêt de Riz, sur lo tronc des arbres; dans le ravin de Charmel, sous les feuilles mortes.

\section{BUIIMUS OBSCURUS.}

IIelix obscura, Müller, Verm. Hist., II, p. 105, 1774. Bulimus obscurus, Draparnaud, Tabl. Moll., p. 63, 1801. et Hist. Moll. France, p. 74, pl. Iv, f. 25, 180 s.

Espèce abondante partout, notamment dans la forêt de Riz, dans le ravin de Charmel; ì Chartèves, à Barzy, etc.

\section{BUIIMUS TRIDENS.}

IIelix tridens, Hüller, Verm. Ilist., II, p. 106, 1774.

Bulimus tridens, Bruguière, Eneycl. méth., Vers ( $\beth^{\circ}$ partie), p. $550,1792$.

Coteaux de Jaulgonne, sous les pierres. 
G. Ferussacia.

\section{FERUSSACIA SUBCYLINDRICA.}

IIelix subcylindricn, Linnơus, Syst. nat. (éd. XII), p.1218, 1767.

Ferussacia subcylindrica, Bourguignal, in Amén. malac., t. I, p. 209, 1856.

Coquille commune dans les prés, au pied des arbres, dans toutes les vallées un tant soit peu humides.

\section{G. CLAUSILIA.}

\section{CIAUSIIIA IAMINATA.}

Turbo laminatus, Montayu, Test. Brit., p. 359, pl. II, f. 4, 1805 .

Clausilia laminata, Turton, Brit. Moll., p. 70, 1850.

Assez abondante au pied des arbres, dans la forêt de Riz et dans le bois de la Thiollerie, a l'onest de Jaulgonne.

\section{CLAUSILIA VENTRICOSA.}

Pupa ventricosa, Drapurmend, Tabl. Moll.. p. (i2, 1801. Clausilia ventricosa, Draparmeul. Ilist. Moll lirance. p. 71, pl. Iv, f. 14, 1803. 


\section{$-26-$}

Sur les trones pourris, sous les vieilles écorees, dans la forêt de Riz.

\section{CTAUSIIIA ROIPUII.}

Clausilia Rolphii, Gray, Mat. arrang. Moll in merl. repos., XV, p. 259, 18501.

Forêt de Riz. Ravin de Charmel. Alluvions du ru d'Argentol.

\section{CLAUSIIIA PITCATUIA.}

Pupa plicatula, Draparnuud, Tabl. Moll., p. (if, 1801 . Clausilıa plicatula, Drapamaud, Hist. Moll. France, p. 72, pl. Iv, f. 17-18, 180 s.

Espece abondante dans les bois, sous les feuilles et au pied des arbres. Forêt de Riz. Ravin de Charmel.

Dans le ravin de la côte le Charmel, à la source de l'Oureq, existe une charmante variété de celte espèce, caractérisée par un test transparent, d'un blanc verdâtre. (Clausilia abina, Benlie, Syn. mélh., p. 52, 1850.)

\section{CIAUSIIIA DRUIDIXICA.}

Clausilia druidilica, Bourguignal. Malac. Brelagne, p. 105, pl. II, f. 5-6, 1860.

Espèce rare. Dans les savards d'Argentol.

\section{CLAUSIIIA NIGRICANS.}

Clansilia nigricans, Je/fieyss, in Linn. Trans., XVI, p. .751, 1828. 
fommune solis lin pierres, sous les mouses, dans les bois et les vallées. - Savards d'A rgentol.

\section{CLAUSTEIA REROUDI.}

Clansilia Reboudi, Dupuy, Hist. Holl. France, f1, 356, tab. xvili, f. 5-4, 1850.

Savards d'Argentol.

\section{CLAUSILIA PARUUIA.}

IIelix parvula, Studer, Faun. Ilelv. in Coxe, Trav. Swilz, Ł. III, p. 431 (sans descriplion), 1789.

Clausilia parvula, Studer, liurz. Ferzeichn, p. 89, 1820.

Espece commune au pied des murs; dans les prós, sous les pierres, sous les écorces des arbres. Vallée de la Marne; le Charmel, Argentol, etc.

\section{G. BALIA.}

\section{BAITA PERVERSA.}

Turbo perversus, Limmens, sisti. nat. (ed. X), I, p. T(iт, 1758.

Balea perversil, Fleming, Brit. anim., 11. 261. 1828.

Balial perversa, Bomrguignal, s. Balia, in Amén. malac.. 2, p. 69, pl. xu1, f. 1-5., $183 ̈ 7$.

Sous l'écorce des vieux arbres an ravin de Charmel. Dans les ruines de Bayard, près de Jaulgonne. 


\section{batia deshayestana.}

Balia Deshayesiana, Bourgnignat, g. Balia, in Amén. malac., 2, p. 74, pl. xıI, f. 4-6, 1857.

Chemin de Bayard.

$$
\text { G. PUPA. }
$$

PUPA SECaLE.

Pupa secale, Draparnaul, Tabl. Moll., p. 59, 1801, et Hist. Moll. France, p. 64, pl. III, f. $49 \cdot 50$, 180 .

Au IIuriot de Barzy. Sous les pierres des coteaux. Alluvions de la Marne.

\section{FUPA DOLIOIUM.}

Bulimus doliolum, Bruguiere, Hist. Vers., in Encycl. méth. ( $2^{\mathrm{e}}$ partie), p. 551, 1792.

Pupa doliolum, Draparnaud, Tabl. Moll., p. 58, 1801. et Hist. Moll. France, p. 62, pl. III, f. 41-42, 180 .

Sous les pierres, dans les endroits un peu ombragés. Collines de Jaulgonne, de Barzy; forêt de Riz.

\section{PUPA MUSCORUM.}

Turbo muscorum, Linnelus, syst. nat. (ide X), I, p. Thi, 1758. 
Pupa muscorum, C. Pfriffer, I)eutsth. Moll, I, p. :5, pl. III, f. 17-18, 1821.

Collines de Jaulgonne, sous les pierres. Alluvions de la Marne, où cette espèce est des plus abondantes.

\section{G. VERTIGO.}

\section{VERTIGO MUSCORUM.}

Pupa muscorum, Draparnaud, Tabl. Moll., p. 56 (exclus. syn.), 1801.

Vertigo muscorum, Michaul, Compl. à Drap., p. 70, 1851.

Sous les pierres, dans les endroits humides, dans les ravins de Charmel et d'Argentol.

\section{VERTIGO EDENTUEA.}

Pupa edentula, Draparnaud, Hist. Moll., p. 59, 1803.

Vertigo edentula, Studer, Syst. Verz., p. 89, 1820.

Dans les alluvions de la Mlarne. - Espèce rare.

\section{VERTIGO MOULINSIANA.}

Pupa Moulinsiana, Dupuy, Catal. extram. Gall. test., $\mathrm{n}^{\circ}$ 284, 1849, et Hist. Moll., p. 415, pl. xx, f. 11 ( $4^{\mathrm{e}}$ fasc.), 1850.

Dans les endroits hunides, sous les pierres. Vallée de la Marne. Forêt de liiz. - liavin d'Argentol. 


\section{VERTIGO ANTIVERTYO.}

Pupa antivertigo, Draparnaud, Tabl. Holl., p. 57, 1801, et Hist. Moll., p. 60, tab. III, f. 52-55, 1805.

Vertigo antivertigo, Michaud, Compl. à Drap., p. 72, 1851 .

Sous les pierres, dans les endroits humides, aux environs de Jaulgonne et de Barzy. - Alluvions de la Marne.

\section{VERTIGO PXGMAE.}

Pupa pygmæa, Draparnand, Tabl. Moll., p. 57, 1801, et Hist. Holl., p. 60, pl. III, f. 50-51, 1805.

Vertigo pygmæa, Férussac, Tabl. syst., p. 68, 1821.

Assez commun sous les pierres. -- Alluvions de la Marne, où il est très-abondant.

\section{VERTIGO VENETZII.}

Vertigo Venetzii, Férussac, Tabl. syst., p. 69, 1821.

Pupa Venetzii, L. Pfeiffer, Symb. ad Ilist. Hel., II, p. $130,1842$.

Espèce rare, ou plutôt difficile à trouver, à cause de son estrême petitesse. Alluvions de la Marne. 


\title{
CRCILIANELLII)A.
}

\author{
G. CACILIANELLA.
}

\section{CECIIIANELIA IIESVIUIEI.}

Circilianella Licsvillei, Bourguignat, Monogr., g. Cxcil., in Amén. malac., I, p. 217, pl. xvı1, f. 6-8, 1868.

Alluvions dı ru de Charmel et de la Marne.

\section{CXCIIIAIEYTA AGIENA.}

Crecilianclla acten?. Bourgnignat, in Amén. malac., t. II, p. 51, pl. I, f. $\overline{3}-4,185 \%$.

Avec la précédente, dans les allurions de la Marne.

\section{AURICULID E。}

\author{
G. CARYGHIUM.
}

\section{CARYCHIUIM IINIMUM.}

Carychium minimum, Maillor, Verm. Mist., II, p. 12\%, 1774. 
Sous les pierres, sous les feuilles, dans les mousses, au pied des arbres, aux environs de Jaulgonne, de Barzy, de Charmel.

\section{CARYCHIUM TRIDENTATUM.}

Saraphia tridentata, Risso, Mist. nat. Nice, t. IV, p. 84, 1826.

Carychium tridentatum, Bourguignat, in Amén. malac., II, p. 14, pl. xv, f. 12-15, 1857.

Alluvions de la Marne.- Forêt de Riz, sous les écorces.

\section{CARYCHIUM STRIOLATUR。}

Carychium striolatum, Boarguignat, in Amén. malac., II, p. 46, pl. x, f. 11-12, 1857 .

Dans les alluvions de la Marne et des ruisseaux.- Peu abondante.

\section{S 2. PULMOBRANCHIATA.}

\section{LIMNEID E.}

G. PLANORBIS.

\section{PLANORBIS CONTORTUS.}

IIelix contorta, Linnaus, Syst. nat. (éd. X), p. 770, 1758. 
Planorbis contortus, Mïller, Verm. IIsist., II, p. 162, 1774 .

La Marne entre Jaulgonne et Barzy.

\section{PLANORBIS CORNEUS.}

Helix cornea, Limeurs, Syst. nat. (éd. X), p. 770, 17 j̈S. Planorbis corneus, Poiret, Coq. Aisne et des environs de Paris, p. 87, 1801 .

Dans la Marne et dans les mares du moulin Launay et d'Argentol.

\section{PLANORBIS COMPLANATUS.}

Helix complanata, Linnous. Syst. nat. (éd. X), I, p. 769 , 1798.

Planorbis complanatus, Studer, Faunul. IIelv. in Coxe, Trav. Switz, III, p. 〈ร๊o, 1789.

Dans les marécages, entre le moulin Launay et Argentol. - Dans la Marne.

\section{PIANORBIS DUBIUS.}

Planorbis dubius, Hartmamn, Wurm. in N.-Alp., I, p. $251, n^{\circ} 119$ B, et Erd und sussw. Gasterop. Schweiz, p. 111, pl. xxxı, 1844, et Bourguignat, Malac. du lac des Quatre-Cantons, p. 4i, pl. I, f. 21-25, 1862 .

Ilabite, avec la précédente, dans la Marne et les ruisseaux fangeux. 


\section{PIANORBIS ROTUNDATUS.}

Planorbis rotundatus, Poiref, Coq. Aisne et des environs de Paris, p. 95, 1801.

Dans la Marne el les ruisseanx.

\section{PLANORBIS VORTEX.}

Planorbis vortex, Müller. Verm. Ilist., II, P. $158,1774$.

Dans la Mlarne et les mares d'Argentol.

\section{PLANOREIS COMPRESSUS.}

Planorbis compressus, Michaud, Compl. à Drap., p. 81, tab. xVI, f. 6-8, 1851 .

Dans les mares, entre le moulin Launay el Argentol.

\section{PLANORBIS ALBUS.}

Planorhis albus, Müller, Verm. Ilist., II, p. 161, 1774. Commune dans la marne et le ruisseau de Jaulgonne.

\section{planotibis crosseanus.}

Planorbis Crosscanus, Bourguiynal, Malac. du lac des Quatre-Cantons, p. 42, pl. I, f. 15-16, 1862. Ascez rare. Dans la Marme, sur les plantes aquatiques. 
PUANORTIS STELMACHZTIUS.

Planorbis stelmachaplius, Bourymignal, Malac. Bretagne, p. 139 , pl. II, f. 10-15, 1860 .

Celte belle espèce, qui vit dans les caux du vallon d'Ar. gentol, a élé décrite de nouveau par II. J. Colbeau, sous lapplation de Planorbis Rofireni (.1m. Soc. malac. de Belgique, I, p. 34, pl. 11, f. 4, 1865).

\section{PIANOFBIS TONEANUS.}

Ilelix fontana, Lightool, in Phil. Trans. LXXXI ( $1^{\text {re }}$ partie), p. 165 , tab. I1, f. 1, 1786.

Planorbis fontanus, Fleming, in Edimb. Encycl., t. VII (1 ${ }^{\text {re }}$ partie), p. $69,1814$.

Dans les mares et les ruisseaux, sous les feuilles des plantes aquatiques.

\section{PMANOREIS IMBRICATUS.}

Planorbis imbricatus, Müller, Verm. Ilisı., II, p. 163̈, 1774.

Alluvions de la Marne.

\section{PLANORBIS CRISTATUS.}

Nautilus crista, Limneus, Syst. nat., p. 709, 1760.

Planorbis cristalus, Dretarnaud. Hist. Moll., [1. "̈, lab. 11, f. 1-3, 1805. 


\section{$-56-$}

Arec l'espece précédente, dans les alluvions de la Marne.

\section{G. PIIYSA.}

\section{RHYSA FONTINALIS.}

Bulla fontinalis, Linne'us, Syst. nat. (éd. X), p. 797, 1758.

Physa fontinalis, Diaparnaud, Tabl. Noll., p. 522, 1801, et Hist. Moll., p. 3̌4, pl. III, f. 8-9, 1803.

Dans les marécages, à Chartèves.

\section{G. AMPIIIPEPLEA.}

\section{AMPHIPEPLEA GIUTINOSA.}

Buccinum glutinosum, Miiller, Verm. Ilist., II, p. 199, 1774.

Amphipeplea glutinosa, Nillsson, Holl. Suec., p. 58, 1822.

La Marne, dans les endroits vaseus et remplis de plantes aquatiques.

$$
\text { G. LININAA. }
$$

IIMNPEA AURICUIARIA.

Helix auricularia, Linnceus, Syst. nat. (éd. X), I, p. 774, $17 \% 8$. 
Limneus auricularius, Draparnaud, Tabl. Moll., p. 48, 1801.

Limnza auricularia, Dupuy, IIist. Moll. France, p. 481 , pl. xxII, f. 8 ( $5^{\mathrm{e}}$ fasc.) 1851.

Échantillons parfaitement caractérisés dans la Marne, sur les tiges des plantes.

\section{IIMN正A CANAIIS。}

Limnara canalis, Villa, in Dupuy, Hist. Moll. France, p. 482, pl. xxir, f. 12 (5e fasc.), 18381.

Dans la Marne, avec l'espèce précédente.

\section{LIMNREA MONNARDI.}

Gulnaria Monnardi, Hartmamn, Erd und sussw. Gasterop. der Schweiz, p. 71, pl. xvm, f. 1-5, 1844.

C'est la première fois que cette belle Limnée, qui semblait spéciale à certains lacs de la Suisse, est constatée en France. Nous l'avons recueillie dans la Marne, où elle paraît rare.

Cette Limnée est caractérisée par une ouverture si développée, que celle-ci dépasse les tours de spire autant en dessus qu'en dessous; de telle sorte qu'elle parait composer entièrement la coquille.

\section{IIMNAEA IIMOSA.}

IIelix limosa, Limnerus, Syst. nat. (éd. X), I, p. 774, 1758. 
Limnæa limosa, Moquin-Tamdon, IIist. Moll. France, II, p. 465, pl. xxxiv, f. 11-12, 1855.

Très-abondante dans tous les ruisseaux. Commune également dans la Marne.

\section{FIMNIFA INTERMEDIA.}

Limnæa intermedia, Lamarcl, An. s. vert., I. VI (2 partie), p. 162, 1822.

Alluvions de la llarne.

\section{IIMNZA STAGINAIS.}

IIelix stagnalis, Linneus, Syst. nat. (éd. X), I, p. 774, 1758.

Limnæa stagnalis, Dupıy, IIist. Moll. France, p. 467, pl. xxII, f. 10 ( $5^{e}$ fasc.), 18 s̆l.

Limnæa stignalis, Bourguigmat, in Amén. malac., p. 94, pil. XII, f. 1-2, 1862 .

Échantillons assez, typiques dans la Iharne, le long des rives de l'illot de Voterre, près de Jaulgonne.

Nous avons, en outre, recueilli, dans la Marne et les rusisseaux, de nombreux individus d'une variété nommée gallica par notre ami J. R. Bourguignat, caractérisée par une columelle moins torilue, moins tronquée, plus épaisse, et dont la callosité, au lieu de se terminer brusquement à fal torsion de la columelle, continue presiue jusqua a la base; enfin par des lours de spire plus ventrus et plus grossièrement striés. 


\section{LIMNAEA PEREGRA.}

Buccinum peregrum, Müller, Verm. Ilist., II, p. 150, 1774 .

limneus pereger, Draparnaud, Tabl. Moll. France, p. 48, 1801, et Hist. Moll., p. 50, pl. 11, f. 54-57, 1803 .

Limnail perenra, Dupuy, Hist. Noll. France, p. 479. pl. xxxı1, f. 6 (5 fase.), 1831.

Dans les alluvions de la Marne.

\section{IIMNEA PATUSTRIS.}

Buccinum palustre, Miiller, Verm. Ilist., II, p. 151, 1774.

Limnae palustris, Fleming, British anim., p. 274, 1828.

Marécages de la vallée d'Argentol. - Alluvions de la Marne.

\section{IIMNIEA GIATRA.}

Buccinum glabrum, Müller, Verm. Ilist., II, p. 183̈, 1774.

Limnara glabra, Dupuy, Hist. Moll., p. 462, tab. xxı, f. 9 (5 fasc.), $183 ̋ 1$.

Échantillons peu Iypiques dans la mare du bois Bocaut, dans la forèt de Iiz. Ces échantillons se rapprochent un pen de la variété gingicula de Goupil /Limnara gingivata, Goupil, Moll. Sarthe, p. (65, pl. 1, f. 8-10, 185̈̈; -Dupuy, Hist. Moll., pl. xxı, f. 9 B, 1831). 


\section{IIMNAA TRUNCATUIA.}

Buccinum truncatulum, Müller, Verm. Hist., II, p. 150 , 1774.

Limnæus truncatulus, Jeffreys, Syst. test., in Trans., Linn., XVI ( $2^{\circ}$ partie), p. 577, 1850.

Limnæa truncatula, Moquin-Tandon, Moll. France, II, p. 475, pl. xxxiv, f. 21-24, 18 כว.

Abondante dans la Marne et les ruisseaux, notamment à l'écluse d'Argentol.

\section{A NCYLIDA. \\ G. ANCYLUS.}

\section{ANCYIUS SIMPIEX.}

Lepas simplex, Buc'hoz, Aldrov. Lothar., p. 256, 1771. Ancylus simplex, Bourguignat, g. Anc., in Journ. conch., IV, p. 187, 1855, et in Spicil. malac., p. 149 , 1862 .

Dans Ia Marne, ainsi que dans le ruisseau de Chartèves, et les étangs Robert et de la Logette.

\section{ANCYIUS GIBBOSUS.}

Ancylus gibbosus, Bourguignat, g. Anc., in Journ. conch., IV, p. 186, 1855 . 
Dans les ruisseamx découlant des fontaines, sur l's pierres.

\section{ANCYIUS IACUSTRIS.}

Patella lacustris, Limnous, Syst. nat. (éd. X), p. 783, 1758.

Ancylus lacustris, Wïller, Verm. Hist., II, p. 199, 1774.

Sur les plantes aquatiques, le long des rives de la Marne. - Espèce très-abondante.

\section{Gasteropomi OPERCULATA.}

\section{$\S 1$. PULMONACEA.}

\section{CYCLOSTOMIDA.}

G. CYCLOSTOMA.

CYCIOSTOMA EIEGANS.

Nerita elegans, Mïller. Verm. Hist., II, p. 117, 177.1. Cyclostoma elegans, Drapamaud, Tabl. Moll., p. 58, 1801, et Hist. Moll., p. 52, tab. I, fig. ち-8, $180 \mathrm{~s}$.

Sur tous les coleaux des environs de Jaulgonne, au pied des lıaies. 


\title{
$-12-$
}

G. ACME.

\section{ACME IINEATA.}

Bulimus lineatus, Draparnaud, Tabl. Moll., p. 67, 1801. Acme lineata, Ilartmamn, in Sturm. Fauna, VI, II. 6, pl. II, 1821.

Sous les mousses et les détritus autour de Jaulgonne. - Espèce rare.

\section{§. BRANCHIATA.}

\author{
PALUDINIDE.
}

G. VIVIPARA.

givipara fasciata.

Nerita fasciala, Mïller, Verm. Hist., II, p. 182, 1774. Viviparia fasciata, Dupuy, Ilist. Moll. France, p. 510 , pl. xxvir, f. 6 (5ั fasc.), $185 \% 1$.

Abondante dans la Marne. 


\title{
G. BYTIINIA.
}

BYTIINIA TENTACUIATA.

Ilelix tentaculata, Linnous, Syst. nat. (éd. X), p. 774, 1758.

Bythinia tentaculata, Stein, Schneck. Berl., p. 92, $18 \% 0$.

Très-commune dans la Marne et les ruisseaux.

\author{
G. PALUDINELLA.
}

\section{PALUDINETIA TURGIDULA.}

Paludinella turgidula, Paladilhe, Nouv. Misc. malac., p. 115 (4 fasc.), 1869.

Espece abondante dans une mire du bois Bocaut, dans la forêt de Riz, ainsi que dans la source de Moulins, près de Jaulgonne.

\section{PALUDINELIA VIRIDIS.}

Bulimus viridis, Poirel, Con. Aisne ct des environs de

$$
\text { Paris, p. 43 (avril), } 1801 .
$$

Paludinella viridis, Frauen/eld, Verzeichn der namen, etc., der gatt. Paludina, p. 109, 1864.

Dans une fontaine, près de Barzy, ansi que dans la sonre salce du bois de Charteves, pris de Jangonne. 


\section{VALVATIDE.}

G. VALVATA.

VALVATA CONTORTA.

Valvata contorta, Nenke, in Zeitschr. für Malak., p. 115, 1845.

Espèce rare. - Alluvions de la llarne.

\section{VATEATA OBTUSA.}

Nerita obtusa, Studer, Faun. Helv. in Coxe, Trav. Schw., t. III, p. 456 (sans caract.), 1789.

Valvata obtusa, Brard, Coq. Paris, p. 190, pl. vi, f. 17, 181 \%.

Excessivement commune dans la Marne, dans les racines des plantes aquatiques.

\section{VAIVATA MINUTA.}

Valvata minuta, Draparnaul, IIist. Moll. France, p. 42, pl. I, f. 56-58, 180 ö.

Alluvions de la Harne. 
vAIVATA CRISTATA.

Valvatiz cristata, Miüler, Verm. IIist., II, p. 198, 1774.

Dans les ruisseaux, ainsi que dans la Marne.

\section{VAIVATA PIANORBUIINA.}

Yalvata planorbulina, Paladilhe, Nouv. Misc. malac. (2 fasc.), p. 49, pl. 111, f. 25-26, 1867.

Habite également dans les ruisseaux, ainsi que dans la Narne.

\section{MOLLUSCA ACEPHALA.}

\section{LAVELLIBRANCIILTA.}

SPIIERIDE.

G. SPHERIUN.

\section{SPHARIUM RIVICOLA.}

Cyclas rivicola, Lamarck, An. s. vert., t.V, p. $388,1818$. 
Spherium rivicola, liourquignat, Amén. mal. in Rev et mag. de zool., $n^{\circ}$ 8, p. 545, 185\%, et Mlonogr. genre Sphærium, p. 12, pl. I, f. 8-12, 1854.

Espèce commune dans la Marne.

\section{SPH正RIUM BOURGUIGNATI.}

Concha ovato-subrhomboidali, subinæquilaterali, compressa, vix tumida, sat fragili, corneo-luteola, eleganter concentrice iprasertim ad partem inferiorem) striatula; - margine superiore convexo; margine inferiore rolundato; antice leviter subtruncato-rotundata; postice magis dilatata, inferius sat producto-subrostrata; - umbonibus antice versis, obsoletis, levigatis, vix tumndulis; - dentıbus cardinalibus exiguis in utrinque valvulis) in formam $\mathrm{V}$ dispositis; - lamellis Jateralibus minimis, parum e'ongatis, in valvula destra) utrinque duplicibus, (iu sinistra) unicis; - ligamento exiguo.

Coquille ovalaire-subrhomboïlale, subinéquilatérale, comprimée, à peine renflée, à test assez fragile, d'une teinte cornéc-jaunatre et éligamment sillonnce de petites striations devenant de plus en plus accentuées vers le bord inférieur. Bord supérienr convese; lord inférieur arrondi. Partie antérieure peu développée, d'une forme arrondic un peu anguleuse. Parlie prstérieure plus développée, légèrement rostriforme. Sommets rejetés en avant, trèsémoussés, linses, à peine renflés. Dents cardinales trèsexiguës se présentant, sur les deus valves, sous la forme d'un $\mathrm{V}$ renversé. Sur la valve droite, Jamelles latéro-postérieure et antérieure, au nombre de deux; sur la valve gauche, une seule lamelle latéro-antérieure et latéro-postérieure. Ligament délicat.

$$
\begin{array}{lrr}
\text { Haut. . . } & \text { 12-14 millim. } \\
\text { Long. . . } & 16-18 & - \\
\text { Epaiss. . } & 7-8 & -
\end{array}
$$


Thans la Marne, il Juulgonne. - Espice peu abondaute.

Nous la possídons égilement de diversess rivières du nord de la france, d'oủ uous l'avons reçue, tantôt comme une variéte trìs compriméc du Spharium corneum, tantôt comme une variété minor, ou un jeune îge du Sphacrium rivicola.

Ce nouveau Spharium, que nous dédions à notre ami MI. Bourguignat, ne peut être rapproché que du Sphærium rivicola.

Notre nouvelle espice se distingue de celle-ci par sa forme rhomboïdale et plus inéquilatérale; par son test comprimé, à peine renflé: par ses striations plus délicates, par ses sommets plus émousés, moins prociminents et plus antírieurs; par ses deuts cardinales en forme de V sur les deux valves; par ses lamelles latéro-postérieure et antérieure plus exiguës, moins allongées, etc.

\section{SPHIRIUM CORIVEUM.}

Tellina cornea, Limanus, Syst. nat. (éd. X), p. (678, 1758.

Sphærium corneum, Scopoli, Intr. ad Hist. nat., p. 598 , 1777.

Les divers échantillons que nous classons sous cette appellation appartiennent, pour la plupart, à la rariété rivalis (Cyclas rivalis).

Dans la Marne el le ruisseau de Jaulgonne.

\section{SPHIERIUII IACUSTRE.}

Tullina lacustris, Vüller, Verm. Ilist., II, p. 201, 1774. 
Sphærium lacustre, Bourguignat, in Amén. malac., t. I, p. 6,1855 .

Ruisseau de Jaulgonne et la Narne, dans les parties vaseuses.

\section{G. PISIDIUM.}

\section{PISIDIUM AMNICUM.}

Tellina amnica, Müller, Verm. Hist., II, p. 20ð, 1774. Pisidium amnicum, Jenyns, Monogr. Cycl. and Pisid. in Trans. Camb. phil. Soc., t. IV ( $2^{c}$ partie), p. 509 , pl. xix, f. 2, 1855.

Assez rare. Dans les alluvions de la Marne.

\section{PISIDIUM CaSERTANUm.}

Cardium casertanum, Poli, Test. utr. Siciliæ, I, p. 65, tab. xv1, f. 1, 1792.

Pisidium casertanum, Bourguignat, Cat. Moll, terr. et fluv. d'Orient, p. 80, 1855.

Dans le ruisseau de Jaulgonne et dans la Jarne.

\section{PISIDIUD PUSIIIUM.}

Tellina pusilla, Gmelin, Syst. nat., 1, p. 5251, 1789.

Pisidium pusillum, Jenyns, Monogr. Cycl. and Pisid. in 
Trans. Camb. phil. Soc., 1. IV (go partie), p. 502, tab. $x x$, f. $4-6,1855$.

Dans-le ruisseau de Jaulgonne.

\section{PISIDIUM NITIDUM.}

Pisidium nilidum, Jemyns, Monogr. Cycl. and Pisid. in Trans. Camb. phil. Soc., t. IV ( $\mathcal{Q}^{\circ}$ partie), p. 504, pl. $x x$, f. $7-8,1855$.

Dans les petits ruisseaux d'Argentol et de Jaulgonne.

\section{PISIDIUM HENSEOWIANUM.}

Tellina IIenslowana, Sheppard, Desc. Brit. shells, in Trans. Linn., t. XIV, p. 149-150, 1825.

Pisidium Ilenslowianum, Jenyns, Ilonogr. Cycl. and Pisid. in Trans. Camb. phil. Soc., 1. IV, p. 508, tab. xxı, f. 6-9, $18 \overline{5}$.

Ilabite dans la Marne.

On rencontre encore dans les alluvions de cette rivière la variété inappendiculata (Bourguignul, in Amén. malac., I, p. 51, 18:4), qui ne diffère du type que par le manque de l'appendice lamelliforme des sommets.

\section{PISIDIUM OBTUSAIE.}

Cyclis obtusalis, Lumuercli, An. s. vert, 1. V, p. 559, 1818. 


\title{
$-50-$
}

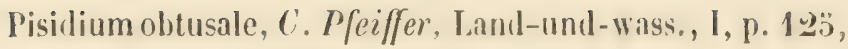
pl. v, f. 21 -22, 1821.

Alluvions de la IIarne.- Assez rare.

\section{UNIONIDE.}

\author{
G. UNIO.
}

\section{UNIO SINUATUS.}

Unio sinuata, Lamarck, An. s. verl., t. VI (1 ${ }^{\text {re }}$ parlic), p. $70,1819$.

Unio sinuatus, Rossmässler, Enum. Un. Lurop., $\mathbf{n}^{\circ} \mathbf{5}$, 1844.

Jans les parties les plus profondes die la Marne.

\section{UNIO RHOMBOIDEUS.}

Nlya rhomboidea, Schroeter, Flussconchyl., p. 186, pl. ॥, f. 5, 1779 .

Unio rhomboideus, Moquin-Tandon, Hist. Moll. France, t. II, p. 568, pl. xLvแ , f. 4-9, $185 \% 3$

Espèce abondante dans la Marne. 
UNIO REQUIENI.

Unio Réquieni, Michaud, Compl. à Drap., p. 106, pl. xvi, f. 24,1851 .

IIabite avec la précédente.

\section{UNIO BATAVUS.}

Mya batava, Maton et Rackelt, Cat. Brit. Lest. in 'Trans. Linn., t. VIII, p. 57, 1807.

Unio batavus, Nillsson, Moll. Succiæ, p. $112,1822$.

Excessivement abondante dans la Marne.

\section{UNIO MANCUS.}

Unio manca, Lamarck, An. s. vert., t. VI (1 ${ }^{\mathrm{re}}$ partic), p. $80,1819$.

Unio mancus, Dupuy, Hist. nat. Moll. France, p. 642

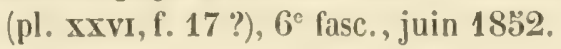

Habile avec la précédente.

\section{UNIO AMNICUS.}

Unio amnicus, Ziegler, in Rossmâssler, Iconogr., III, p. 31 , pl. xv, f. $212,1856$.

Très-commune dans la Marne. 


\section{$-82-$}

\section{G. ANOIOONTA.}

\section{ANODONTA ARENARIA.}

Hya arenaria, Schroeter, Flussconchyl., p. 165, pl. II, f. 1, 1779 .

Anodonta arenaria, Bourguigmal, Malac. de la Bretagne, p. 78,1860 .

Dans les parties vaseuses, convertes de roseaus, de la Marne.

\section{ANODONTA OBLONGA.}

Anodonta oblonga, Millet, in Mém. Soc. agr. Angers, I, p. 242, pl.11, f. 1, 1851 .

Jans la Marne.

\section{ANODONTA ANATINA.}

Mytilus anatinus, Limnens, Syst. nil. (id. X), I, P. 706. 1758 .

Anodonta anatina, Lamarcli. An. s. vert., t. VI (I" par(ie), p. $85,1819$.

Habite avec la précédente.

\section{ANODONTA DUPUYI.}

Anodonta Dupuyi, J. Ruyy. Dese. nouv. Anod. in Rev. de 
mag. zool., I, p. 29, pl. 1 et ir, 18:19, et Dupuy, Hist. Moll. France, p. 606, tab. XviI, f. 15, 1852.

Dans les endroits vaseux de la Marne.

\title{
DREISSENIDE.
}

\author{
G. DREISSENA.
}

\section{DREISSENA FIUVIATILIS.}

IIytilus (polymorphus) fluviatilis, Pollas, Vuy. en Russie, Append., p. 211, 1771.

Dreissena fluviatilis, Bourguignat, Imén. malac., I, p. $16 \overline{5}, 1855$.

Espèce abondante dans la Narne. 





\section{CATALOGUE}

InIs

\section{HOLLLSOULS TERRRESTRES BT FLIVIITILES}

OBSERVÉS

A UX ENVIRONS DE JAULGONNE (Aisne)

PAR

MIM. CH. LALLEMANT ET G. SERVAIN.

AOUT 1869.

Haris,

IMPRISERTE FT LIBRAIRIE INE UI" Ve BOUCHARI)-IIUZARI,

RUE DE L'ÉPERON, 5.

$\overline{1869}$ 



\section{EN VENTE}

\section{CIIEZ SAVY, 2\%, RUE HAUTEFEUILLE}

E:T

CHEZ BOUCHARD-HUZZARD, 5, RUE DE L'ĖPERON.

D. DUPUY (l'ablí). Mémoire sur les coquilles terrestres de Bernède, près de Lectoure. Paris, 1869 , in-8; avec pl.

G. DUTAILIY, Descriptions de quelques espèces nouvelles du groupe de l'Helix Raspaili. Paris, 1867, in-8.

LEFOURNEUX. Catalogue des mollusciues terrestres et fluviatiles recueillis dans le département de la Vendée et particulièrement dans l'arrondissement de Fontenay-le-Comte. I'aris, 1869, in-8.

J. MABILLE. Archives malacologiques. In-8. - $1^{\text {r }}$ a $5^{\circ}$ fasc., 1869. - (Sous presse le $6^{\text {e }}$ fasc.)

- Histoire des mollusques terrestres et fluviatiles du bassin parisien. $1 \mathrm{vol}$. in-8, avec pl. 11 , ou color. (Sous presse la $1^{\text {re }}$ partic.)

P. A. IMOITESSIER. Histoire malacologique du département de l'Hérault. Paris, 1868. 1 vol. in-8 avec 1 pl. (En vente chez Bouchand-Huzard à Paris, el chez Coulet, libraire, à Montpellier.)

PALADILHE (le doct.). INouvelles miscellanées mala-

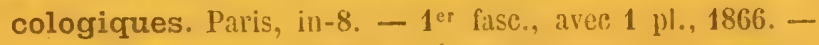
$2^{\mathrm{e}}$ fasc., avec $2 \mathrm{pl}, 1867 .-3^{\mathrm{e}}$ fasc., avec 1 pl., 1868. (Sous presse le $4^{\mathrm{c}}$ fasc.)

SAINT-SINON (Alfied DE). Descriptions d'espèces nouvelles du genre Pomatias, suivies d'un aperçu synonymique sur les espèces de ce genre. Paris, 1869 , in-8.

G. SERVAIN. Malacologie des environs d'Ems et de la vallée de la Lahn. Paris, 1869 , in-8.

Paris. - Iuprimerie de madame couve liombliarl-lluzard, rue de l'Fiperon, $5,-1860$. 





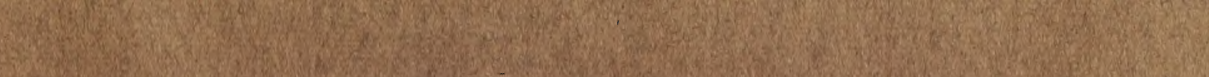

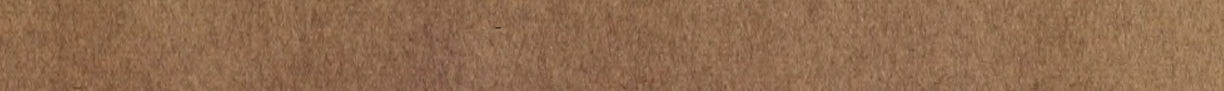
2.5. 20.5. S6.

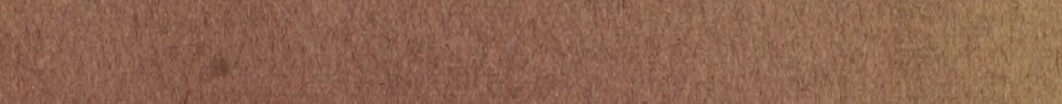

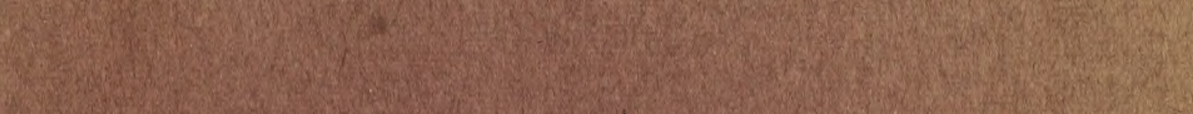

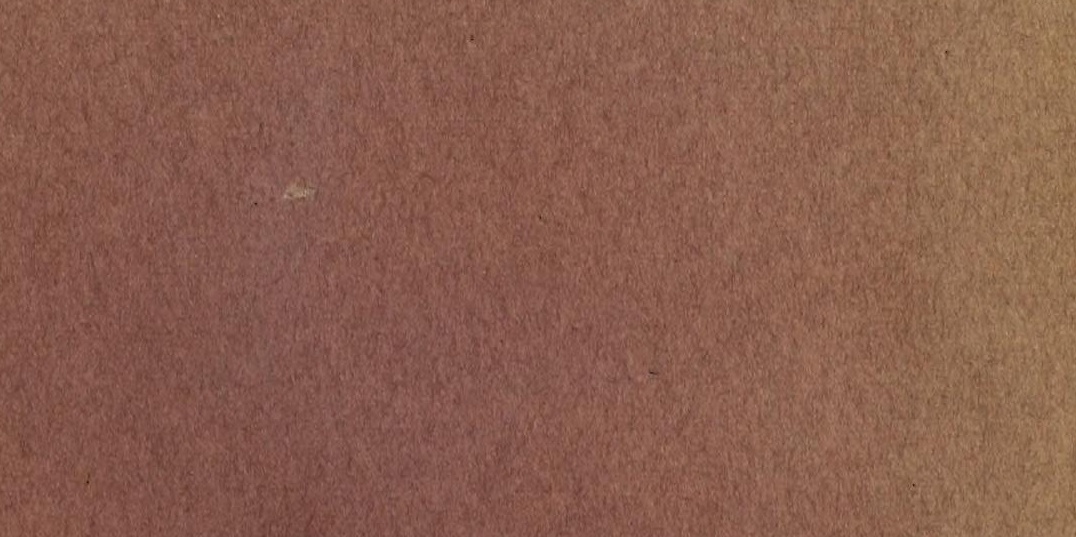

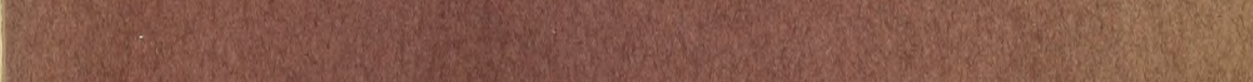




